\title{
IJCARS - IPCAI 2020 special issue: 11th conference on information processing for computer-assisted interventions - part 1
}

\author{
Stamatia Giannarou ${ }^{1}$ - Ilker Hacihaliloglu ${ }^{2}$
}

Published online: 25 May 2020

(c) CARS 2020

For its 11 th edition, it is a great pleasure to present this special issue of IJCARS - international conference on information processing for computer-assisted interventions (IPCAI). In light of the ongoing COVID-19 pandemic, the IPCAI 2020 Conference Organizing team and the IPCAI steering committee have recommended to hold the IPCAI 2020 annual meeting planned for June 23-24, 2020, in Munich, Germany, in conjunction with the Computer Assisted Radiology and Surgery (CARS) Congress, as a virtual conference.

With a first edition in 2010, the IPCAI meeting has continued to provide a leading forum to discuss the latest developments in computer-assisted interventions (CAI). With a strong emphasis on research and practice, CAI focuses on computer-based tools and methodologies to support medical interventions with the overall objective to enable precise and safe systems for minimally invasive medical interventions. Hence, the domain is heavily anchored in bringing forward novel developments in enhanced planning, realtime imaging, instrument guidance and visualization, as well as situation awareness and cognition. To promote translational research, the IPCAI meetings seek to showcase papers presenting novel technical algorithms and theory, clinical applications and hardware as well as software systems and their validation.

One differentiating aspect of IPCAI is that submitted manuscripts are expected to be of journal quality, with the goal to publish all accepted manuscripts in this special volume of the International Journal of Computer Assisted Radiology and Surgery (IJCARS). To this end, the IPCAI review process was rigorous and involved evaluation of each

Stamatia Giannarou

stamatia.giannarou@imperial.ac.uk

Ilker Hacihaliloglu

ilker.hac@soe.rutgers.edu

1 Hamlyn Centre for Robotic Surgery, Imperial College London, Bessemer Building, South Kensington Campus, SW7 2AZ London, UK

2 Department of Biomedical Engineering, Rutgers University, 599 Taylor Road, Piscataway, NJ 08854, USA manuscript by two Area Chairs and at least three external reviewers. Authors were able to provide a revised version of their manuscript and a response to reviewers based on the reviewers' comments. The revised manuscripts were subject to an additional review process, ensuring that all reviewers' critiques were properly addressed and that the quality was appropriate for journal publication. Prior to acceptance, manuscripts were discussed by the Program Chairs, Program Board members and the Conference Chairs.

For this year's edition, IPCAI received 79 manuscript submissions, spanning a strong geographic representation from Europe, North America and Asia. In total, 39 manuscripts were accepted for presentation at IPCAI 2020. Accepted manuscripts reflected breadth of key topics in CAI and include: (i) Interventional imaging; (ii) Surgical data science; (iii) Tracking and navigation; (iv) Interventional imaging; (v) Investigational robotics, evaluation and validation; (vi) Interventional ultrasound; and (vii) Surgical planning, simulation and advanced intraoperative visualization.

For the second time, following the success in the 2019 meeting, the CAI community was invited to submit long abstracts to showcase their work. Emphasis was given on presenting (i) exciting new breakthroughs relevant to IPCAI and (ii) novel ideas with preliminary but limited experimental validation. A total of 9 submissions were received, of which 4 were accepted for presentations at IPCAI 2020. These submissions were reviewed by the Program Chairs and General Chairs.

As in previous years, the 2020 IPCAI meeting is designed to encourage interactions between attendees and allow time for constructive discussion. All authors of accepted manuscripts are invited to present their work in an initial short 5-min video presentation and live Q\&A sessions by the authors on the paper itself. The conference attendees will also be asked to vote for papers that they would like to nominate for the "Audience Choice Awards". Accepted long abstract submissions will be showcased by short 5-min video presentations as well as live Q\&A sessions by the authors on the paper itself. This year's program also includes a keynote 
talk, sponsor booths as well as discussion and social gathering sessions.

We would like to take this opportunity to thank the Area Chairs, the Program Board and all of the reviewers for their dedication to maintaining the high quality of IPCAI and for selecting outstanding manuscripts that will no doubt stimulate exciting discussions at the meeting.

We acknowledge our generous sponsors for their continued support over the years that has enabled us to recognize both IPCAI authors and reviewers for their much-deserved dedication and scientific enthusiasm through several paper awards. The awards include the Outstanding Reviewer Award, Audience Choice Awards, Bench-to-Bedside Award, Translational AI/ Deep Learning award and Best Paper Award, all made possible through their generous sponsorship. On behalf of all awardees, we are very grateful for this continued support!
We also thank all of the CARS Congress Organizers and Editorial Office for their continued help and support with logistics, as well as with the IJCARS manuscript management and review process.

Perhaps most importantly, we are grateful to all authors for choosing to submit their manuscripts to IPCAI and hope that the readers enjoy this special issue. The continued success of IPCAI demands our ongoing commitment.

Stamatia Giannarou, Ilker Hacihaliloglu (Program Chairs)

Publisher's Note Springer Nature remains neutral with regard to jurisdictional claims in published maps and institutional affiliations. 